\title{
45
}

LA-UR- 08-76

Approved for public release;

distribution is unlimited.

Title: A metamaterial solid-state terahertz phase modulator

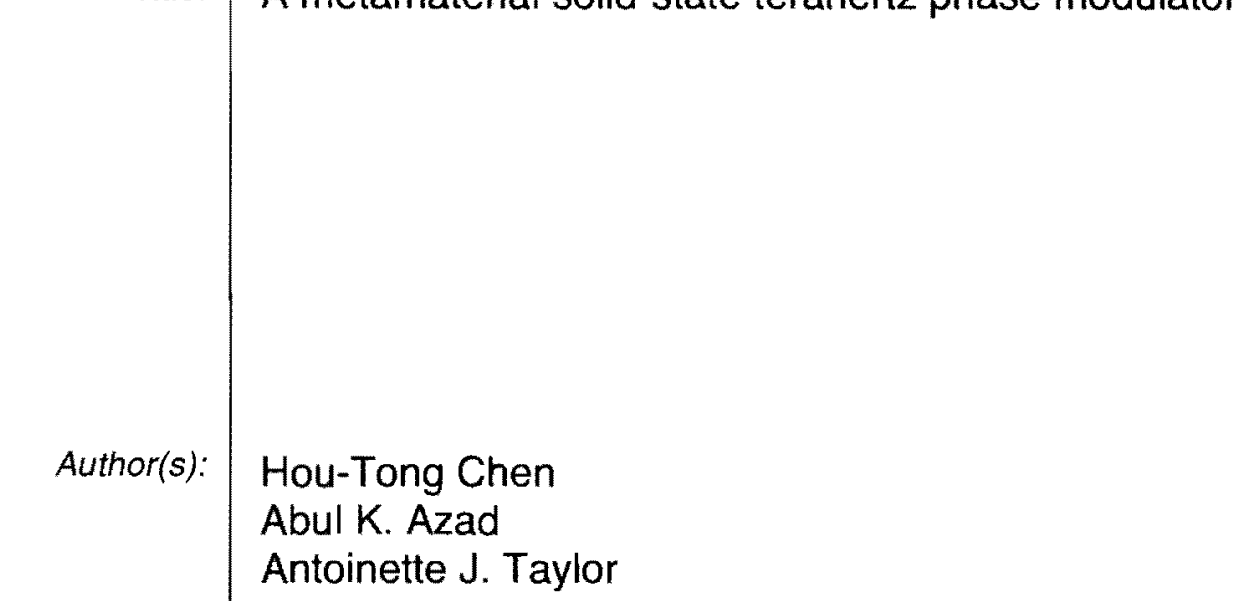

Intended for: Nature Photonics

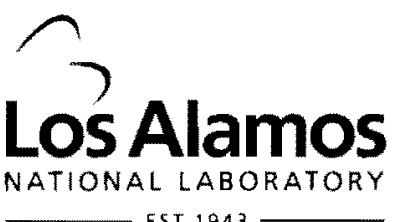

EST. 1943

Los Alamos National Laboratory, an affirmative action/equal opportunity employer, is operated by the Los Alamos National Security, LLC for the National Nuclear Security Administration of the U.S. Department of Energy under contract DE-AC52 06NA25396. By acceptance of this article, the publisher recognizes that the U.S. Government retains a nonexclusive, royalty-free license to publish or reproduce the published form of this contribution, or to allow others to do so, for U.S. Government purposes. Los Alamos National Laboratory requests that the publisher identify this article as work performed under the auspices of the U.S. Department of Energy. Los Alamos National Laboratory strongly supports academic freedom and a researcher's right to publish; as an institution, however, the Laboratory does not endorse the viewpoint of a publication or guarantee its technical correctness. 


\section{A metamaterial solid-state terahertz phase modulator}

Hou-Tong Chen ${ }^{*}$, Willie J. Padilla ${ }^{2}$, Michael J. Cich ${ }^{3}$, Abul K. Azad', Richard D. Averitt ${ }^{4}$ and Antoinette J. Taylor ${ }^{1}$

${ }^{\prime}$ Center for Integrated Nanotechnologies, Materials Physics \& Applications Division, Los Alamos National Laboratory, Los Alamos, New Mexico 87545, USA

${ }^{2}$ Department of Physics, Boston College, 140 Commonwealth Avenue, Chestmut Hill, Massachusetts 02467, USA

${ }^{3}$ Sandia National Laboratories, MS1085, Albuquerque, New Mexico 87185, USA

${ }^{4}$ Department of Physics, Boston University, 590 Commonwealth Avenue, Boston, Massachusetts 02215, USA

"e-mail: chenhr@lanl.gor

During the past two decades terahertz time-domain spectroscopy ${ }^{1}$ and quantum cascade lasers ${ }^{2,3}$ represent two of the most important developments in terahertz science and technology. These technologies may help to contribute to many proposed terahertz applications and global efforts are currently underway ${ }^{4}$. However, the necessary devices and components for effective control and manipulation of terahertz radiation require substantial development beyond what has been accomplished to date. Here we demonstrate an electrically controlled planar hybrid metamaterial device that is able to control the phase of terahertz radiation with constant insertion loss over a narrow frequency band. Alternatively, the metamaterial device may 
operate as a terahertz broadband modulator ${ }^{5}$. As verification to the versatility of our device utilizing the interrelated phase control and amplitude switching, we perform terahertz time-domain spectroscopy with our hybrid metamaterial modulator replacing a commercial mechanical optical chopper, and demonstrate similar broadband performance.

The controllable electromagnetic properties arising from engineered metamaterials facilitate novel opportunities for manipulating electromagnetic radiation ${ }^{6}$. This electromagnetic response originates from resonances in patterned metallic subwavelength structures in an array to form an artificial material. Although natural material response is somewhat rare at terahertz frequencies, metamaterials may be scaled to appropriate dimensions to yield significant terahertz electromagnetic response that is the basis for the construction of novel devices operating in this regime. In addition to the emerging electromagnetic phenomena achieved by metamaterials such as negative index of refraction $^{7,8}$, super-resolution in optical imaging ${ }^{9.10}$ and electromagnetic invisibility ${ }^{11}$, terahertz metamaterial devices have set state-of-the-art performance for many components such as frequency-agile far-infrared filters ${ }^{12,13}$, all-optical switches and modulators ${ }^{14,15}$, and perfect absorbers ${ }^{16.17}$. In particular, room temperature, electrically driven metamaterial devices consisting of only a single unit cell layer in the propagation direction have been demonstrated with high speed and modulation depth ${ }^{5,18}$.

Despite the rapid progress in THz science and technology, a component much sought after but still largely unavailable, is an efficient high performance terahertz phase shifter. Its counterparts in the microwave and optical regimes have found various important applications, for example, phased array antenna systems and high-speed Mach-Zehnder modulators. However these are, in general, difficult to extend to operation within the 
terahertz regime. There have been a few efforts to demonstrate terahertz phase shifters, but they suffer from problems such as the operation temperature and speed. For instance, terahertz phase shifters using semiconductor quantum well structures operate at cryogenic temperatures $^{19,20}$, and liquid crystal based terahertz phase shifters are inherently very low speed $^{21}$, thus severely limiting their applications. The single layer planar hybrid metamaterial device demonstrated here operates at room temperature, and is capable of shifting the phase of terahertz transmission by as much as $\sim \pi / 6$ radians through the application of a bias voltage of 16 volts.

A single unit cell of the device is illustrated schematically in Fig. la. Metallic electric split-ring resonators $(\mathrm{SRRs})^{22}$ are periodically patterned to form a square array and connected to each other by metal wires. They are fabricated on a $1 \mu \mathrm{m}$ thick epitaxial $n$ doped GaAs layer with an electron density of $2 \times 10^{16} \mathrm{~cm}^{-3}$ grown on an intrinsic GaAs wafer. The SRRs and semiconductor form the Schottky diode structure that can, upon application of an external voltage, actively modify the depletion zone. The ability to control the carrier density in this depletion zone permits tuning of the local dielectric properties near the gap of a SRR thereby altering its resonant properties. This further results in changes to the terahertz transmission (amplitude, phase, or both) of the metamaterial device. In the design shown here, the SRR gaps are located at the four outer corners so that they are directly exposed to the ohmic contact through the $n$-doped semiconductor substrate layer. This maximizes depletion of the charge carriers near the metamaterial gaps upon application of bias voltage - essential to the control of the metamaterial resonances. The micro-fabrication of the device has been described previously ${ }^{5}$ and an optical microscopy image is shown in Fig. 1b. 
At a voltage bias of 16 volts the metamaterial device yields two electrical resonances at $0.81 \mathrm{THz}$ and $1.7 \mathrm{THz}$ shown in Fig. 2a, driven by the linearly polarized terahertz electric field with normal incidence presented in Fig. 1b. The resonance at $0.81 \mathrm{THz}$ is from the individual SRRs and due to the inductive-capacitive coupling of the circulating currents (Fig. 1c), while the resonance at $1.7 \mathrm{THz}$ originates from a collective dipolar resonance (Fig. 1d), in which the resonance frequency is also dependent on the periodicity of the SRRs array ${ }^{23}$. At zero voltage bias the resonances are rather weak due to the conducting substrate that shunts the metamaterial capacitance thereby significantly damping the response. Under a reverse voltage bias of 16 volts an increase in depletion occurs, thus reducing the damping causing an increase in oscillator strength for both resonances. Note that the depletion near the split gaps plays a critical role in the recovery of resonances, as illustrated in Figs. 1c and d of the resonant surface current density. The main resonant loss occurs at the split gaps across which currents can flow in the undepleted SRRs.

In comparison to previously published results on metamaterial switches ${ }^{5}$, the device presented here yields a higher modulation index of the terahertz transmission amplitude. Under a reverse voltage bias of 16 volts, the amplitude of the transmitted terahertz electric field at $0.81 \mathrm{THz}$ decreases from $t_{\mathrm{OFF}}=0.56$ to $t_{\mathrm{ON}}=0.25$, a change of $\Delta t=55 \%$ (intensity change of $\Delta T=80 \%$ ), as shown in Fig. 2 a. Here "OFF" and "ON" indicate the metamaterial resonances are switched off and on, respectively. The terahertz transmission amplitude at $1.7 \mathrm{THz}$ decreases as well from $t_{\mathrm{OFF}}=0.48$ to $t_{\mathrm{ON}}=0.30$. Additionally, between the two resonances the reverse voltage bias significantly increases the terahertz transmission amplitude.

Switching of the metamaterial device presented here yields another important desired functionality - phase shifting of terahertz radiation. As shown in Fig. $2 \mathrm{~b}$, at $0.886 \mathrm{THz}$ the 
phase of terahertz transmission is $\phi_{\mathrm{ON}}=-0.51 \mathrm{rad}$ under the reverse voltage bias of 16 volts as compared to $\phi_{\mathrm{OFF}}=0.05 \mathrm{rad}$ with no voltage bias, resulting in a shift of $\Delta \phi=0.56 \mathrm{rad}$. The measured phase shift occurs over a bandwidth of $\sim 23 \mathrm{GHz}$ (i.e. $0.880-0.903 \mathrm{THz}$ ) with a change in amplitude of less that $10 \%$ over this range. It should be noted that this phase change occurs within a single metamaterial unit cell in the propagation direction. One may then construct a multi-layer phase shifter from designs presented here to easily realize the ultimate goal of a $2 \pi$ phase shifter. Additionally, at the operational frequency of the phase shifter (indicated by the vertical lines in Fig. 2), the terahertz transmission amplitude is near $60 \%$ (without consideration of the substrate insertion loss) and almost independent on the applied voltage bias. This is advantageous since this terahertz phase shifter can operate with reasonably high terahertz transmission amplitude. It should be noted that substrate insertion loss could be lowered by the addition of a metamaterial impedancematched layer to both the incoming and outgoing surfaces of the device ${ }^{24}$.

The use of a semiconductor as the metamaterial substrate makes it readily integrable with other solid-state terahertz sources and detectors, which may result in very compact terahertz systems for various applications. In addition, various schemes would permit addressing of individual SRR elements, thus permitting application of, for example, a voltage gradient to be applied to the array. This would permit real time beam steering or lensing and other manipulations of a terahertz radiation thereby enabling applications such as active personnel screening in airports, or allowing for a terahertz beam from a moving satellite to be locked to a particular receiver. For such potential applications, it is important to characterize the phase shift as a function of the applied bias voltage. Figure $2 \mathrm{c}$ displays the results revealing a linear phase shift as a function of the bias voltage from 0 to 14 volts at $0.89 \mathrm{THz}$. A slight saturation at higher voltages occurs because the resonance is almost 
totally restored, and because of a reduction in the Schottky resistance resulting from the increasing leaking currents.

We now turn toward demonstration of the metamaterial device functioning as a terahertz modulator and quantitatively compare it to a routinely used commercial modulator, i.e. a mechanical optical chopper, in a terahertz time-domain spectroscopic system. Figure 2 indicates that both the amplitude switching and phase shifting in our terahertz metamaterial device are inherently narrow band - a result of the resonant nature of metamaterials. However, as shown in Fig. 2, the terahertz transmission amplitude and phase are not independent of each other, but are related by Kramers-Kronig (KK) relations ${ }^{25}$. Near frequencies where the amplitude has no strong dependence on the applied voltage bias, the phase experiences a maximum shift, and vice versa. Thus, although both amplitude and phase maxima are narrowband, the KK relations indicate that the metamaterial depicted in Fig. 1a will modulate over an extended range - a result of the causal relation between amplitude and phase. This property indicates that the device can be used as a broadband modulator, as is often used in terahertz time-domain spectroscopic systems. The frequency dependent modulation of terahertz transmission coefficient is given by

$$
|\Delta \tilde{t}(\omega)|=\left|t_{\mathrm{OFF}}(\omega) e^{i \phi_{\mathrm{OFF}}(\omega)}-t_{\mathrm{ON}}(\omega) e^{i \phi_{\mathrm{ON}}(\omega)}\right|
$$

where both the amplitude and phase contribute to the modulation. Figure $3 a$ presents the experimental results of the metamaterial device acting as a broadband modulator. The timedomain waveform of the terahertz modulation (differential) signal upon application of a square ac voltage bias alternating between 0 and 16 volts is shown as the red curve in Fig. 3a. Here the mechanical optical chopper has been removed from the regular terahertz time- 
domain spectroscopic system. As a reference, the terahertz transmission signal through a bare GaAs substrate was also measured by terahertz time-domain spectroscopy. The modulated terahertz spectrum produced by the metamaterial device is shown in Fig. $3 b$ with a broadband and rather flat response of $|\Delta t(\omega)| \approx 0.3$ between $0.8 \mathrm{THz}$ and $1.7 \mathrm{THz}$, beyond which the terahertz radiation is also modulated but with decreasing modulation depth. Note that these frequencies are the frequencies of the two metamaterial resonances, as indicated in Fig. 2. Thus by utilizing known methods capable of increasing the frequency span between the two metamaterial resonances ${ }^{26}$, it is possible to further broaden the range of modulation to match the range covered by terahertz time-domain spectrometers.

Here we would like to emphasize that our metamaterial modulator directly modulates terahertz waves. This is an important distinction since alternate methods yield modulation during the terahertz generation process, i.e. modulated sources ${ }^{27,28,29}$. Thus our modulator is a true "terahertz component", in the sense that it can be added to various terahertz sources such as backward wave oscillators, terahertz quantum cascade lasers, etc. The metamaterial broadband terahertz modulator can be immediately implemented into a terahertz timedomain spectrometer replacing the typical mechanical optical chopper. In order to compare the performance of our metamaterial modulator to that of a mechanical optical chopper (both operating at $377 \mathrm{~Hz}$ ), we plot in Fig. 4a the division of two subsequent transmission measurements, a so-called 100\% line. This quantity then represents the frequency dependent noise of the system as deviations from 100\%, thus indicating the usable range. We take as our evaluation point deviations of $\pm 2.5 \%$, indicated by the gray area of the top portion of Fig. 4. The mechanical optical chopper yields a range from $93 \mathrm{GHz}$ to $2.32 \mathrm{THz}$, while the metamaterial modulator achieves a range from $161 \mathrm{GHz}$ to $1.88 \mathrm{THz}$. Thus comparing similar noise levels in the two modulations, the mechanical optical chopper yields approximately $30 \%$ more bandwidth in this particular terahertz system. The signal- 
to-noise ratio is still not comparable since the mechanical optical chopper can achieve $100 \%$ modulation depth with no insertion loss. However, for first generation terahertz metamaterial modulator no performance optimization has been attempted. There are many paths available for improvement, such as fabricating an impedance-matched metamaterial layer on both the incoming and outgoing surfaces, or utilizing different aspect ratio designs for the unit cell that to increase the effective bandwidth.

We conclude by utilizing our metamaterial device as the modulator operating in a THz time-domain system to measure the complex transmission through a second metamaterial sample fabricated on intrinsic GaAs substrate, in which the unit cell is shown in the inset to Fig. 4b. The transmitted terahertz amplitude spectrum is shown in Fig. 4b, clearly identifying the metamaterial resonance near $1 \mathrm{THz}$. As before, we compare our experimental data to that measured by a regular terahertz time-domain spectrometer using a mechanical optical chopper. The good agreement apparent in Fig. 4 confirms the applicability of this metamaterial modulator and evinces the potential of metamaterials, although consisting of only a single planar layer, to function as devices. We further note that, unlike mechanical optical choppers, which are limited to modulation speeds of kilohertz, the metamaterial modulator has been demonstrated to operate up to $2 \mathrm{MHz}$. Faster modulation speeds equate to improvements in system performance, e.g. shorter acquisition times, higher signal-to-noise, etc. Figure $4 \mathrm{~b}$ also shows the results at $30 \mathrm{kHz}$ which show comparable performance to the results obtained at $377 \mathrm{~Hz}$.

In conclusion, we have demonstrated a single-layer, electrically controllable terahertz metamaterial phase shifter yielding $\Delta \phi=0.56$ radians with constant insertion loss. The large number of metamaterial elements may be individually controlled and monolithically fabricated, indicating the potential use for more complex terahertz devices such as 
electrically steerable arrays for active terahertz beam steering and lensing. The amplitude modulation demonstrated here yields an improvement over the existing state-of-the-art by $50 \%$. This metamaterial device has been shown to modulate a complex transmission signal and we demonstrated its use as a broadband modulator in a terahertz time-domain spectrometer.

Acknowledgements We thank Igal Brener of SNL for coordinating the sample fabrication, and John O'Hara for discussions and the use of the terahertz system. We acknowledge support from the Los Alamos National Laboratory LDRD Program. This work was performed, in part, at the Center for Integrated Nanotechnologies, a US Department of Energy, Office of Basic Energy Sciences Nanoscale Science Research Center operated jointly by Los Alamos and Sandia National Laboratories. Los Alamos National Laboratory, an affirmative action/equal opportunity employer, is operated by Los Alamos National Security, LLC, for the National Nuclear Security Administration of the US Department of Energy under contract DE-AC52-06NA25396.

Author information Reprints and permission information is available online at http://npg.nature.com/reprintsandpermissions/. Correspondence and requests for materials should be addressed to H.T.C.

Competing financial interests The authors declare that they have no competing financial interests. 


\section{Figure legends}

Figure 1 Design of electrically driven terahertz metamaterial phase shifter. a, Schematic of device structure of one unit cell and its cross section indicating the operation principle. b, Optical microscopy image of the active area of the device with gold thickness $200 \mathrm{~nm}$, line width $4 \mu \mathrm{m}$, split gap spacing $2 \mu \mathrm{m}$, out dimension $36 \mu \mathrm{m}$, and period $50 \mu \mathrm{m}$. The polarization of the normally incident terahertz radiation is also indicated. $\mathbf{c}$ and $\mathbf{d}$, Numerical simulations of surface current density excited from the inductive-capactive and collective dipolar resonances at 0.81 and $1.7 \mathrm{THz}$, respectively.

Figure 2 Terahertz transmission spectra through the metamaterial device. a, Transmission amplitude, and $\mathbf{b}$, phase spectra at 0 volts and reverse bias voltages of 4 and 16 volts, respectively. The vertical line indicates that at frequencies with large phase shifting but the amplitude is unchanged. c, Voltage dependence of the phase shift at $0.89 \mathrm{THz}$.

Figure 3 Broadband modulation of terahertz radiation. a, Time-domain measurements of the terahertz modulation (differential) signal when the device is under a square voltage bias alternating between 0 and 16 volts. The reference terahertz signal is measured through a bare substrate using the regular terahertz time-domain system with an optical chopper. b, After Fourier transformation, the complex modulation in frequency domain is divided by the reference so that the modulation amplitude spectrum is obtained. In the frequency range roughly between the two resonances, the terahertz signal is most effectively modulated. 
Figure 4 Terahertz time-domain spectroscopy using the broadband metamaterial modulator. a, Amplitude spectral ratio of two subsequent measurements using the metamaterial device or a mechanical optical chopper as the modulator in the terahertz time-domain system. The gray area indicates the $\pm 2.5 \%$ noise deviation. $\mathbf{b}$, Terahertz transmission amplitude spectra through a metamaterial sample (unit cell shown in the inset) using the metamaterial modulator $(377 \mathrm{~Hz}$ and $30 \mathrm{kHz})$ or a mechanical chopper $(377 \mathrm{~Hz})$. 


\section{References}

${ }^{1}$ Mittleman, D. Sensing with terahertz radiation, Springer-Verlag, Berlin, 2003.

${ }^{2}$ Köhler, R. et al. Terahertz semiconductor-heterostructure lasers. Nature. 417, 156-159 (2002).

${ }^{3}$ Williams, B. S. Terahertz quantum-cascade lasers. Nature Photon. 1, 517-525 (2007).

${ }^{4}$ Tonouchi, M. Cutting-edge terahertz technology. Nature Photon. 1, 97-105 (2007).

${ }^{5}$ Chen, H.-T. et al. Active terahertz metamaterial devices. Nature 444, 597-600 (2006).

${ }^{6}$ Smith, D. R. Pendry, J. B. \& Wiltshire, M. C. K. Metamaterials and negative refractive index. Science 305, 788-792 (2004).

${ }^{7}$ Veselago, V. G. The electrodynamics of substances with simultaneously negative values of $\varepsilon$ and $\mu$. Sov. Phys. Usp. 10, 509-514 (1968).

${ }^{8}$ Shelby, R. A., Smith, D. R. \& Schultz, S. Experimental verification of a negative index of refraction. Science 292, 77-79 (2001).

${ }^{9}$ Pendry, J. B. Negative refraction makes a perfect lens. Phys. Rev. Lett. 85, 3966-3969 $(2000)$

${ }^{10}$ Fang, N., Lee, H., Sun, C. \& Zhang, X. Sub-diffraction-limited optical imaging with a silver superlens. Science 308, 534-537 (2005).

${ }^{11}$ Schurig, D. et al. Metamaterial electromagnetic cloak at microwave frequencies. Science 314, 977-980(2006).

${ }^{12}$ Chen, H.-T. et al. Complementary planar terahertz metamaterials. Opt. Express 15, 10841095 (2007). 
${ }^{13}$ Chen, H.-T. et al. Experimental demonstration of frequency-agile terahertz metamaterials. Nature Photon. 2, 295-298 (2008).

${ }^{14}$ Padilla, W. J., Taylor, A. J., Highstrete, C., Lee, M. \& Averitt, R. D. Dynamical electric and magnetic metamaterial response at terahertz frequencies. Phys. Rev. Lett. 96, 107401 (2006).

${ }^{15}$ Chen, H.-T. et al. Ultrafast optical switching of terahertz metamaterials fabricated on ErAs/GaAs nanoisland superlattices. Opt. Lett. 32, 1620-1622 (2007).

${ }^{16}$ Landy, N. I. Sajuyigbe, S. Mock, J. J. Smith, D. R. \& Padilla, W. J. Perfect Metamaterial Absorber. Phys. Rev. Lett. 100, 207402 (2008).

${ }^{17}$ Tao, H. et al. A metamaterial absorber for the terahertz regime: Design, fabrication and characterization. Opt. Express 16, 7181-7188 (2008).

${ }^{18}$ Chen, H.-T. et al. Hybrid metamaterials enable fast electrical modulation of freely propagating terahertz waves. Appl. Phys. Lett. 93, 091117 (2008),

${ }^{19}$ Libon, I. H. et al. An optically controllable terahertz filter. Appl. Phys. Lett. 76, 2821$2823(2000)$.

${ }^{20}$ Kersting, R., Strasser, G., Unterrainer, K. Terahertz phase modulator. Electron. Lett. 36, $1156-1158(2000)$.

${ }^{21}$ Hsieh, C.-F. Pan, R.-P. Tang, T.-T. Chen, H.-L. \& Pan, C.-L. Voltage-controlled liquidcrystal terahertz phase shifter and quarter-wave plate. Opt. Lett. 31, 1112-1114 (2006).

${ }^{22}$ Padilla, W. J. Aronsson, M. T. Highstrete, C. Lee, M. Taylor A. J. \& Averitt, R. D. Electrically resonant terahertz metamaterials: Theoretical and experimental investigations. Phys. Rev. B 75, 041102R (2007). 
${ }^{23}$ Acuna, G. Heucke, S. F. Kuchler, F. Chen, H.-T. Taylor, A. J. \& Kersting R. Surface plasmons in terahertz metamaterials. Opt. Express 16, 18745-18751 (2008).

${ }^{24}$ Lee, J. W. et al. Invisible plasmonic meta-materials through impedance matching to vacuum. Opt. Express 13, $10681-10687$ (2005).

${ }^{25}$ Jackson, J. D. Classical electrodynamics. 3rd Edition. John Wiley \& Sons, New York, 1998.

${ }^{26}$ Azad, A. K. Taylor, A. J. Smirnova, E. \& O'Hara, J. F. Characterization and analysis of terahertz metamaterials based on rectangular split-ring resonators. Appl. Phys. Lett. 92, $011119(2008)$.

${ }^{27}$ Zhao, G. Schouten, R. N. van der Valk, N. Wenckebach, W. Th. \& Planken, P. C. M. Design and performance of a $\mathrm{THz}$ emission and detection setup based on a semi-insulating GaAs emitter. Rev. Sci. Instrum. 73, 1715-1719 (2002).

${ }^{28}$ Shen, Y. C. Upadhya, P. C. Linfield, E. H. Beere, H. E. \& Davies, A. G. Ultrabroadband terahertz radiation from low-temperature-grown GaAs photoconductive emitters. Appl. Phys. Lett. 83, 3117-3119(2003).

${ }^{29}$ Cai, Y. et al. Coherent terahertz radiation detection: Direct comparison between freespace electro-optic sampling and antenna detection. Appl. Phys. Lett. 73, 444-446 (1998). 


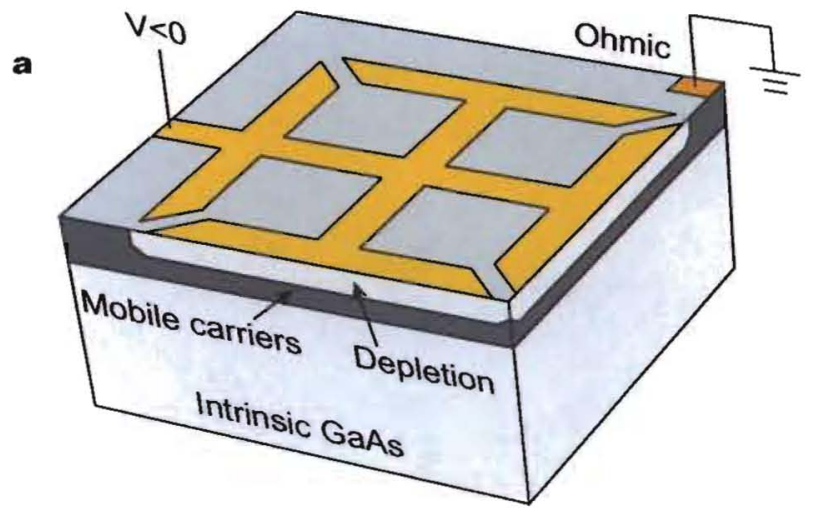

b

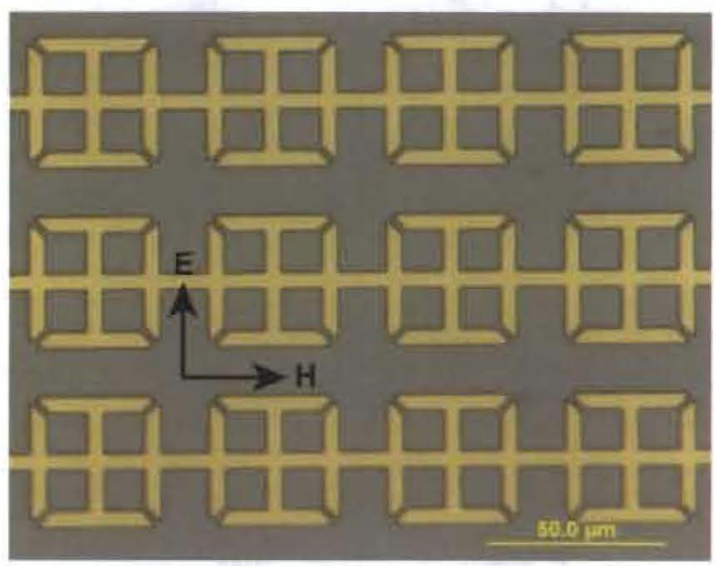

c

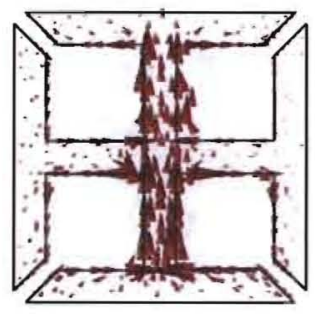

d

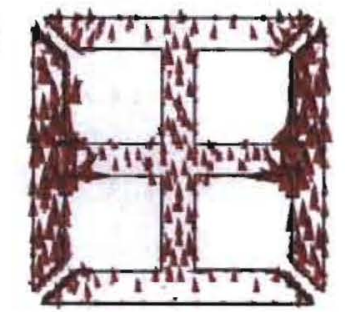



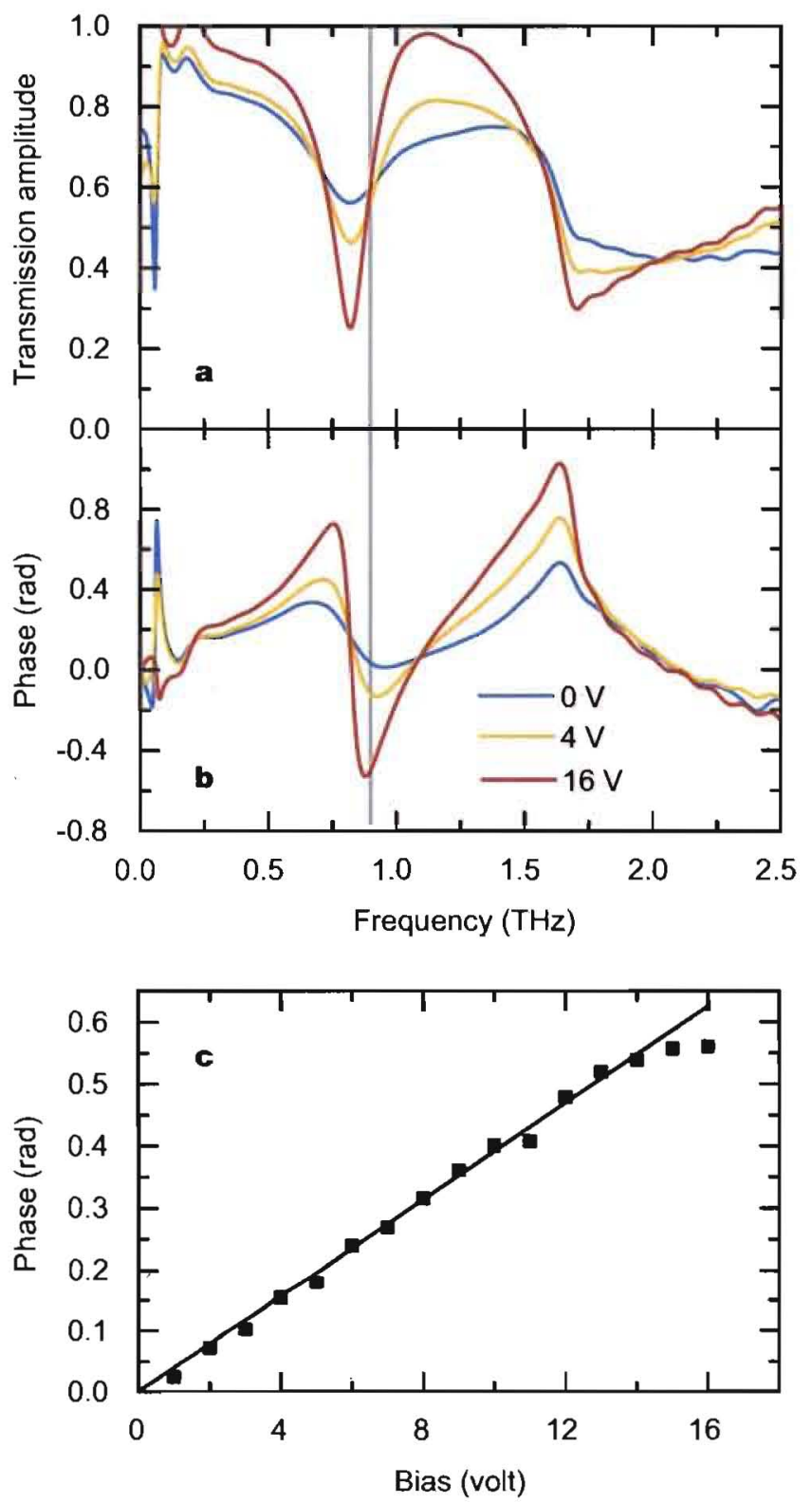

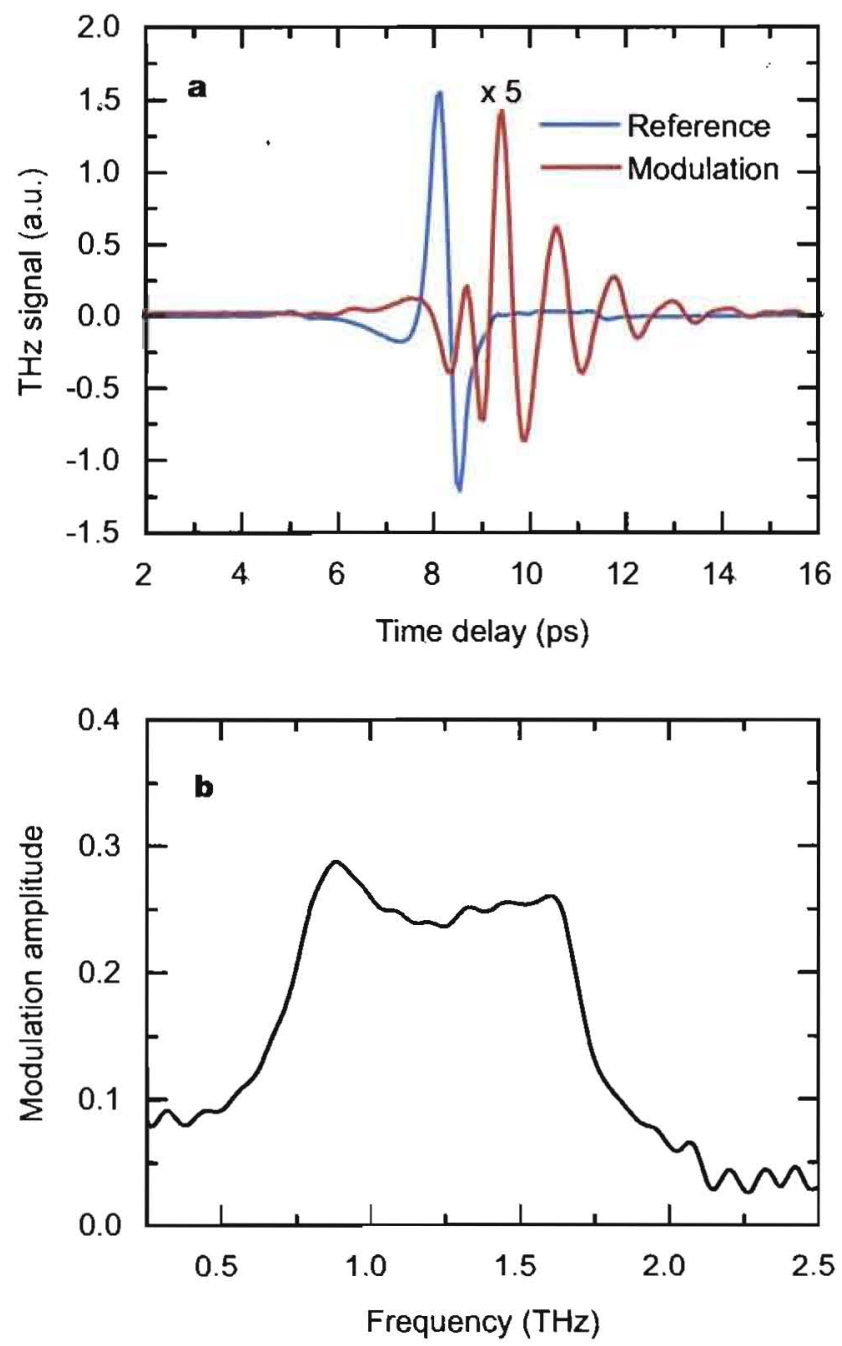


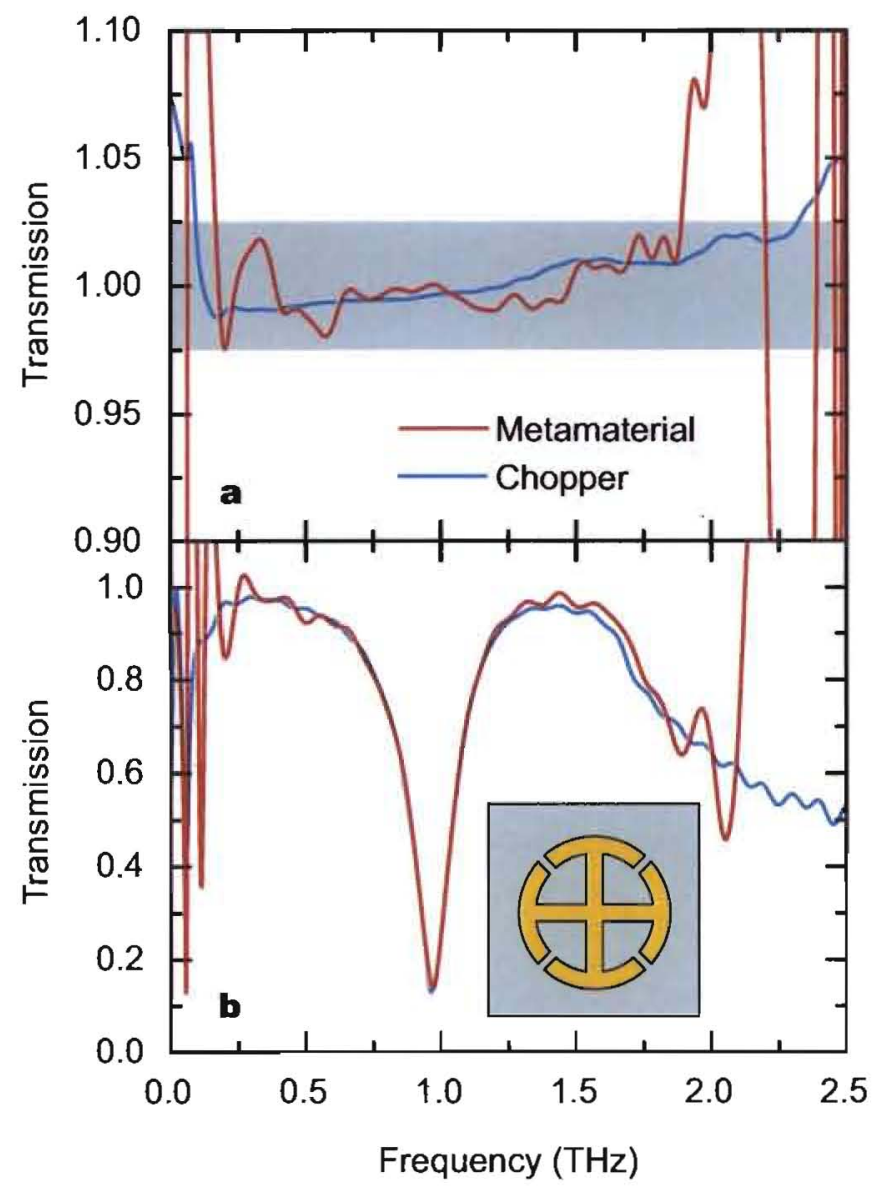

